\title{
Quelles idées les rédacteurs des « guides pratiques pour minitel » se font-ils des usages et des usagers ?
}

What conception do the authors of "Practical Guides for Videotex" have of common practice and their readers?

\section{François Poulle}

\section{OpenEdition}

\section{Journals}

Édition électronique

URL : http://journals.openedition.org/edc/2794

DOI : $10.4000 /$ edc. 2794

ISSN : 2101-0366

\section{Éditeur}

Université Lille-3

\section{Édition imprimée}

Date de publication : 1 mai 1991

Pagination : 81-94

ISSN : 1270-6841

\section{Référence électronique}

François Poulle, «Quelles idées les rédacteurs des « guides pratiques pour minitel » se font-ils des usages et des usagers? », Études de communication [En ligne], 12| 1991, mis en ligne le 15 janvier 2012, consulté le 19 avril 2019. URL : http://journals.openedition.org/edc/2794 ; DOI : 10.4000/ edc. 2794

Ce document a été généré automatiquement le 19 avril 2019

(C) Tous droits réservés 


\section{Quelles idées les rédacteurs des « guides pratiques pour minitel » se font-ils des usages et des usagers?}

What conception do the authors of " Practical Guides for Videotex " have of common practice and their readers?

François Poulle

1 On trouve, à la bibliothèque du CNET d'Issy les Moulineaux ; rangés côte à côte sur une étagère six ouvrages qui se ressemblent par le sujet traité, le genre littéraire et même le titrage. A peu de chose près ils se dénomment tous « guide pratique », entendent guider les pas du lecteur dans l'utilisation du minitel et se sont succédés à un rythme qui peut laisser rêveur plus d'un auteur à succès. De 1983 à 1987, à six reprises, des éditeurs ont engagé des rédacteurs, investi de l'argent et espéré trouver des acheteurs pour un conseil que d'une part l'administration des Télécoms donnait gratuitement et dont d'autre part la nécessité ne s'imposait pas d'emblée : l'utilisation du minitel. Que se passe-t-il avec cet appareil ? Se demande le chercheur tombé en arrêt devant ces six guides côte à côte alignés. Ce modeste boitier connu pour la rusticité de son usage (ne suis-je pas parvenu à le faire utiliser par ma vieille maman) dissimulerait-il une dimension cachée ou des usages inédits? Les craintes de la population de ce pays devant les technologies modernes sont-elles si aiguës qu'elles garantissent le succès commercial sur un marché pourtant réputé difficile, celui du guide pratique?

2 Car, pour avoir à certaines occasions vendu sa plume à quelques éditeurs opérant sur ce marché, le chercheur sait qu'au pays du bricolage les guides pratiques parviennent dificilement à trouver leur public. Qu'ils se présentent en qualité de prescripteur d'emploi (construisez seul votre cheminée) ou comme manuels de dépannage (par exemple dans l'automobile) le succès n'est jamais rapide. Ce n'est que lentement que l'idée de recourir à un guide pour telle ou telle activité chemine dans l'esprit du public. Il s'écoule donc un temps relativement long avant qu'une activité soit en mesure d'apporter 
la renbabilité ou même les bénéfices à ses guides pratiques. Ces six éditions en cinq ans alertent donc la curiosité de notre chercheur. Il s'apprête à ouvrir l'un d'entre eux pour chercher les clés d'une pareille réussite.

3 Mais un scrupule l'arrête alors. Dans un recoin de son cerveau, n'est-il pas en train d'intenter un procès d'intention à des éditeurs, voire à des auteurs ? N'est-il pas en train de se demander si les motivations éditoriales à propos de ces guides pratiques ont bien été la recherche du marché en tant que tel. C'est-à-dire la mise en circulation d'ouvrages destinés à être acquis par des abonnés au téléphone trouvant insuffisant leur maniement du minitel. Ou bien si une autre logique n'a pas guidé les pas des éditeurs. Une logique selon laquelle l'existence ou l'inexistence de marché, la rentabilité ou la non rentabilité de leurs publications, le succès commercial ou la mévente seraient indifférents. Dans cette seconde logique les éditeurs auraient reçu des " aides à publication " qui leur auraient permis d'échapper à la loi du marché. Et la motivation de ceux qui auraient accordé ces aides n'aurait pas tant été de vendre du conseil que de faire connaître un produit (le minitel) en faisant semblant d'en faciliter l'usage. De la publicité clandestine en quelque sorte.

Or, les soupçons étant une chose et l'administration de la preuve une autre, cette logique éditoriale perverse ne peut se contenter d'être énoncée. Elle doit être prouvée. Pour ce faire, il faudrait que le chercheur connaisse les tirages, les prix de revient et les chiffres de vente de chacun de ces guides. Et encore! De tels renseignements, à supposer qu'ils soient accessibles, ne fourniraient qu'insuffisamment d'explications. Les stratégies éditoriales outrepassent souvent la simple logique économique. Un éditeur peut accepter de perdre de l'argent sur un produit si cette perte lui permet de prendre pied sur un créneau. Il ne contrevient pas pour autant à la déontologie éditoriale.

5 L'hypothèse d'une sorte d'opération de promotion occulte, qui a mis en éveil la curiosité de notre chercheur, ne peut, faute des instruments adéquats, être développée dans l'espace de l'économie éditoriale. Mais elle reste tapie dans un coin de l'esprit du chercheur comme le soupçon que Iago glisse à l'oreille d'Otello. Elle murmure, insidieuse : « ces guides pratiques sont-ils vraiment des guides? A qui s'adressent-ils? Qui donc est assez bête pour dépenser les trente ou quarante francs, prix conseillé en quatrième de couverture de l'un de ces guides, pour s'entendre dire qu'il faut brancher un minitel à une prise de courant avant de s'en servir ? De quels usages est-il donc question ici ? Fouille, chercheur, gratte, investigue ».

6 Le chercheur emprunte donc les six guides à leur étagère et les emmène sur sa petite table. Là, il lit, il fouille, il gratte, il analyse.

7 Que cherche-t-il ? Dans l'organisation générale des rubriques, comme dans l'argumentation interne de chacune de ces rubriques, il cherche à repérer la trace d'une " commande » qui serait tout à la fois présente et absente. Ou si l'on préfère les marques d'un " écart ». Quelque chose qui parle du trouble du rédacteur à gages partagé entre la faiblesse du discours qu'il est autorisé à tenir et la tentation d'approches qu'il lui serait préférable d'éviter.

8 Après un survol rapide, cette oscillation peut déjà être identifiée au niveau de la représentation que les rédacteurs se font des utilisations du minitel. Les automobiles servent principalement à rouler. La plupart des guides pratiques qui en traitent sont unanimes sur ce point ; ils n'envisagent ni l'automobile comme manifestation de la personnalité de son propriétaire, ni son bricolage comme règlement des scènes de 
ménage. A quoi servent les minitels ? A offrir des informations rédigées ailleurs ou à produire/mettre en circulation des informations produites par son usager ? Bien qu'ils s'adressent à des cibles distinctes les six guides soit ne distinguent guère entre rédaction et réception soit entremêlent les deux. Et, partant de là, ils ne se décident pas à trancher entre un minitel complétant la panoplie des robots électro-ménagers et un minitel/ machine à communiquer.

\section{Un corpus de six guides pratiques.}

9 - 1983, CECOD, Voyage au pays du Minitel.

- 1984, Scraen, Dominique et Rochas, Sibylle, Minitel mode d'emploi, PSI.

- 1984, David, Jacques, Minitel, votre guide pratique, Cedic Nathan.

- 1985, Saboureau, Jean Pierre et Bouche, Geneviève, Guide pratique du videotexte et du minitel, Cedic Nathan.

- 1986, Bazin, Dorothée et Doré, Monique, Informatex, guide du minitel dans les entreprises. Hommes et données.

- 1987, Doré, Dominique et Fuzeau, Pierre, Vie pratique en Minitel, Borneman.

$\mathrm{Du}$ point de vue des cibles décelables, le plus ancien (CECOD) s'adresse à des professionnels du commerce et de la distribution. Les deux récents (Informatex et Vie pratique en minitel) se présentent comme le découplement d'une même démarche en fonction des cibles visées : les entreprises pour le premier, le grand public pour le second. Avec un rédacteur commun aux deux.

Les trois guides de $84 / 85$ visent le grand public.

11 Quant à la structure des sommaires, on note de fortes différences entre le plus ancien et le plus récent. Le premier, celui du CECOD, est très succinct en matière de prescription d'utilisations. Il s'en tient surtout à des pétitions de principe et des allez-y voir. En revanche, Informatex, l'un des plus récents, est presque un annuaire des services. Ces différences s'expliquent par l'évolution des pratiques sociales elles-mêmes. Les guides $84 / 85$ consacrent une dizaine de pages chacun à expliquer dans quels genres de lieux on peut se procurer un minitel et comment le brancher. Tandis que « Vie pratique en minitel "(1987) fait l'économie de ce type de rubrique. Le grand public est censé savoir désormais à quoi s'en tenir.

12 Néanmoins ces six guides partagent une stratégie de communication commune. Celle-là même qui fonde le principe de tout guide pratique quelle que soit la branche d'activité couverte et qui consiste à laisser entendre au lecteur que les rédacteurs se situent à ses côtés pour l'accompagner dans sa démarche d'appropriation. Ils ont connu avant lui les mêmes difficultés, voire les mêmes angoisses. Grâce à des explications, ils l'aideront à vaincre les inhibitions et résoudre les incertitudes. Cette stratégie explicite fonde l'homogénéité de ce corpus de six guides.

\section{Structure des trois guides grand public.}

13 D'autant plus que trois d'entre eux, ceux destinés au grand public des années $84 / 85$, présentent entre eux d'encore plus fortes ressemblances. Matériellement ils se présentent tous trois sous forme d'une reliure cartonnée d'un format presque carré, 
contenant une quarantaine de pages et présentant des rubriques sensiblement comparables. par trois idées évidemment non explicitées mais plus ou moins sous-jacentes :

- La comparaison avec des techniques d'information concurrentes.

Dans le cas d'Informatex le minitel est mis en comparaison avec la presse écrite, la radio ou SVP. Dans celui de Vie pratique les catalogues de vente par correspondance sont désignés comme concurrents.

- L'esquisse d'une observation des performances effectives en termes de relations du type qualité/prix. Utilisation de critères comme la garantie de mise à jour, la compétitivité dans la disponibilité...

- L'esquisse aussi d'une évaluation des itinéraires cognitifs que chaque service propose ou requiert de son usager. Et donc d'une sorte de vécu social en termes d'attentes, d'efficacité informationnelle ( « les dessins complètent l'information » note Vie pratique à propos d'un serveur), et de navigabilité.

Dans les deux cas donc un discours s'engage. Un discours où le rédacteur d'un guide pratique se met à la place de l'usager non seulement pour mettre le minitel en service ou en indiquer les modes de tarifiction. Mais aussi pour avoir précédé l'usager sur des attentes qui ne concernent plus seulement l'appareil en tant que tel mais aussi les 
services auxquels il permet d'accéder. Le rédacteur se met en posture de conseiller sur les bonnes et les mauvaises adresses à la manière d'un guide gastronomique ou touristique.

Or cette démarche appropriatrice appelle les remarques suivantes :

- Vis-à-vis des guides antérieurs. On conçoit bien que c'est là le type de rubrique qui aurait pu suivre les vingt premières pages d'initiations (accès à l'annuaire) et donc prendre la place des «topos télématiques » dans les plans types des guides $84 / 85$. Ce qui revient à dire que ces topos ont fonctionné à titre de bouche trous, pour faire le poids de pages. Oui sans doute. Mais pourquoi cette démarche appropriatrice a-t-elle attendu 1986 pour se manifester ? Était-ce parce que les dits services n'existaient pas encore ? Ou parce que les moyens conceptuels et théoriques d'une investigation de ce type faisaient alors défaut?

- Dans les deux guides $86 / 87$, si cette démarche appropriatrice se manifeste, elle ne le fait pas pour autant de façon cohérente. Du fait qu'ici deux pages - pour Informatex - et là cinq - pour Vie pratique - s'inspirent de ce type de démarche, il ne s'en déduit pas pour autant que les rubriques qui suivent - dans les deux cas repérage, description et expérimentations de serveurs - procèdent du même esprit. Bien au contraire. Après avoir évoqué le cas de figure de cet industriel partant pour la Finlande et en mal d'informations clés, Informatex tourne court et s'engage dans un répertoire de " services ", qui est une simple liste d'adresses. Autant dire qu'aucun industriel partant pour la Finlande n'est prêt à s'aventurer dans cette jungle de codes d'accès dont rien n'indique s'ils mènent à des culs de sac ou à des informations pertinentes et actualisées. Un guide Michelin, dont l'acheteur sait que le prix de vente est fonction d'une démarche évaluatrice en amont, n'est pas un annuaire du téléphone.

De la même façon l'analyse multicritères que propose "Vie pratique " n'est pas davantage mise en pratique dans le répertoire des services qui constitue l'essentiel du guide. A titre d'exception, le service SNCF est blâmé pour sa lenteur ${ }^{1}$. Mais des critères comme la navigabilité ou l'entropie proposés au lecteur à la page 37 sont oubliés lorsqu'il s'agit d'évaluer concrètement des services.

Dans les deux guides la démarche, qui envisagerait de partir du besoin en informations d'un individu lambda et d'examiner la pertinence, les réponses télématiques existantes, n'est pas menée. Dans le cas de l'industriel si des services téléphoniques comme SVP existent c'est justement parce que, le rassemblement d'informations exigeant du temps et une spécialisation, il est plus économique de demander et de payer que de chercher soimême. Seul un serveur télématique fonctionnant sur le même principe que SVP implanté soit dans l'entreprise soit extérieurement - peut représenter une alternative concurrentielle au problème posé. Or cette notion d'alternative concurrentielle n'est pas envisagée. Comme il est impossible qu'elle soit ignorée des rédacteurs ${ }^{2}$, il faut s'interroger sur les raisons qui les amènent à faire tantôt état de pertinence, tantôt à rompre avec celle-ci. Est-ce l'absence de moyens conceptuels et théoriques ou la malignité ?

\section{L'hypothèse du complot.}

Or il se trouve que notre chercheur met la main, quelques étagères plus loin, sur une étude commandée par la DOT au Centre de Formation des Journalistes (Ouéry 1983). Cette étude sur " l'écriture et la mise en page télématique » considère la mise en page comme l'anticipation d'une appropriation. Elle comporte les comptes rendus de tests opérés sur 
des usages témoin. Elle prend le parti du producteur de sens ultime (le lecteur) et de sa recherche de plaisir et d'efficacité.

L'étude de Guéry postule le minitel non comme un vulgaire appareil électro-ménager qu'il suffit de brancher mais comme une machine à communiquer. Une machine qui produit du sens. Cette étude fait donc une distinction entre l'utilisation (le bon fonctionnement technique) et l'usage social : la rentabilité dans l'appropriation du sens.

D'autre part, à l'intérieur au sein même de cette démarche appropriatrice, elle fournit une grille d'évaluation des critères d'usages à partir de laquelle peuvent se bâtir des guides pratiques.

En d'autres termes les deux concepts clés dont on a constaté l'absence dans les guides pratiques avaient pourtant été énoncés antérieurement à leur rédaction.

Par ailleurs, il est difficile de croire que cette étude ait été ignorée par les rédacteurs des guides. Ceux-ci semblent avoir été recrutés dans le vivier de matière grise qui prospère dans les environs de la DOT. Le nom de certains d'entre eux se retrouve sur des intitulés d'études. Et d'ailleurs cet organisme public a parrainé officiellement au moins l'un de ces guides puisqu'une préface est signée, ès qualités, par un ingénieur des Télécoms.

Le thème du complot, ou plus précisément de la publicité clandestine, revient donc tarauder l'esprit du chercheur. Il ne s'agit plus de rechercher les preuves d'un achat d'espace public contraire à la déontologie éditoriale. Mais de s'interroger sur cette confusion, qui ne saurait être innocente, entre utilisation et usage. Ne traduit-elle pas un dévoiement volontaire de l'esprit du service public. Car à qui profite le crime ? Qui gagne de l'argent en disant aux usagers branchez-vous, connectez-vous et vous verrez ce que vous verrez ? Qui a tout à gagner à ne pas éduquer le public à un usage économique du minitel ? Les Télécoms parbleu.

30 C'est trop facile, on favorise (avec ou sans aides financières) la publication de guides pratiques qui d'une part familiarisent le public avec le phénomène télématique grand public mais qui surtout, parce que c'est du livre, donc théoriquement du produit intellectuellement non servile, laissent entendre que le phénomène que l'on veut faire naître est déjà né. Donc on falsifie de l'offre pour pouvoir se dire ensuite débordé par les demandes.

31 A cela on ajoute un flou théorique qui ne peut que favoriser la consommation télématique. Les défilements de pages inutiles, les petits dessins qui prennent un temps fou à venir sur l'écran et ne servent à rien, les navigations exaspérantes... tout cela qui fait enrager l'usager réjouit les Télécoms. D'autant plus que l'acte d'achat en télématique ne peut être comparé à ce qu'il est dans ce monde auquel il ressemble par un commun maniement de caractères alphabétiques : le monde du livre et de la presse écrite. Dans ce monde, une sorte de contrat implicite lie l'éditeur à l'acheteur/lecteur. Grâce aux codes d'emballage (titrage, quatrième de couverture, couleurs, grammage du papier), l'éditeur fait un certain nombre de promesses quant à la matière informative qu'il offre à la vente. L'acheteur met en relation ces promesses avec le prix et prend sa décision. S'il est déçu il peut jeter le journal ou le livre et se dire qu'on ne l'y reprendra plus mais quoiqu'il en soit il a construit sa décision d'achat à partir d'un contrat clairement énoncé.

32 En télématique par contre, du fait que la facturation est temporelle, l'usager/acheteur a l'impression de s'enfoncer dans une jungle. Lorsqu'il se rend compte que l'information qu'il recherche va lui coûter plus cher en argent et en temps qu'il n'est prêt à y mettre et encore savez-vous vraiment combien vous déboursez chaque fois que vous consultez ? - 
il est déjà trop engagé pour pouvoir reculer. Les termes du contrat éditorial sont soumis à une évolution sur laquelle il n'a pas de contrôle.

De ce fait, le marché télématique réclame effectivement des guides pratiques. L'usager serait prêt à payer le prix d'un livre pour que quelqu'un l'ait précédé dans les arcanes des serveurs et lui dise : attention ici cul de sac. Or, comme on l'a vu plus haut, aucun des guides étudiés ne remplit exactement cette fonction.

Dans l'hypothèse du complot, on suppose que c'est en connaissance de cause que des responsables de services publics mettent en circulation - ou encouragent à la publication - des ouvrages qui prétendent remplir une fonction tout en ne la remplissant pas. Dans la littérature exotique et coloniale que dévore parfois notre chercheur, ce type d'opérations que mènent les chefs de rezzous s'appelle : « empoisonner les puits ».

\section{L'hypothèse de l'impensé théorique.}

Intellectuellement, les thèses qui font appel à des complots sont reposantes. Une fois les méchants démasqués on se sent toujours mieux. Malheureusement, comme n'importe quelle assertion, les complots doivent être prouvés. Dans notre cas, toute la démonstration repose sur l'affirmation selon laquelle les rédacteurs de guide auraient disposé d'un matériel théorique approprié à une évaluation des usages et auraient refusé d'y recourir. Or cette supposition pêche par inexactitude. Le matériel théorique ne peut être considéré comme véritablement complet et aisément disponible pour des vulgarisations. Ainsi l'étude du CFP\} (Guéry 1983) si elle est effectivement organisée autour du concept d'appropriation d'un sens (et non de mise en marche d'un appareil) ne développe pas cette appropriation au-delà de l'espace de la page. En bon journaliste, Guéry sait évaluer les parcours cognitifs de l'oeil du lecteur à l'intérieur d'une page une fois qu'elle est formée sur l'écran, mais son étude reste muette sur la dimension dynamique de la télématique :

- Le chaînage de pages et donc les vitesses d'appropriation.

- La navigation à l'intérieur des arborescences et donc la nécessité de l'invention de syntaxes de navigation sans commune mesure avec les codes de la presse écrite.

- La production d'informations par l'usager (écritures des choix ou des mots-clés) et donc l'instauration d'une relation qui évoque davantage la conversation que la lecture.

Par ailleurs il serait réconfortant de penser que ces trois principales lacunes sont imputables à l'ancienneté de l'étude du CFPJ. En 1983 c'étaient les premiers pas de la télématique ; depuis la réflexion sur l'usage a fait d'importants progrès. Or il n'en est rien. Quelques centimètres plus loin sur la même étagère notre chercheur trouve une étude relativement récente, celle que la DBMIST a commandée au bureau d'études FUTUR SIMPLE en 1987. C'est une enquête menée sur 19 services universitaires qui croise deux démarches: d'une part un questionnaire auprès de responsables de services et d'autre part un relevé des temps de connection. Il s'y ajoute une très modeste incursion sur quelques serveurs à manière d'une évaluation d'usage. Dans ce dernier domaine, l'étude de Guéry, toute ancienne qu'elle soit, n'est pas dépassée.

Certes, l'étude de Futur Simple esquisse bien une logique de l'appropriation lorsqu'elle propose (pp. 22-23) de distinguer entre les "Services tous publics » dits "à logique administrative » et les " services plus ciblés [...] dans lesquels les informations sont structurées selon une logique plus éditoriale ». De même elle essaye de croiser les chiffres 
de connection avec les offres de menus (type de rubriques). Mais ce croisement s'arrête très vite. Il ne va pas jusqu'à de réelles investigations dans des services où seraient observées non seulement les offres nominales mais aussi les navigations réelles (vitesse d'accès, lisibilité, entropie des codes).

A titre indicatif on trouve quelque part dans cette étude une remarque sur les messageries : les rédacteurs s'interrogent sur la faible utilisation de celles-ci par les usagers étudiants et ils se demandent si ces messageries « correspondent bien à un besoin ». Mais ils se gardent bien de s'interroger sur la logique du « besoin » et notamment sur la relation de pertinence ou d'impertinence que cette logique du besoin entretient avec la logique de l'offre. A supposer que j'ai « besoin » de quelque chose, si ce qui m'est offert par tel service ne m'appète pas j'irai satisfaire mon besoin autrement et ailleurs.

En fait dans cette étude de FUTUR SIMPLE on retrouve le même mécanisme intellectuel que dans les modes d'emploi : sous couvert d'études de l'usage social ce qui est observé ce sont des offres. Et ces offres sont examinées du point de vue des émetteurs et non du point de vue des usagers.

Les lacunes de la réflexion sur les usages sociaux ne sont donc pas une exclusivité - et donc une perversité - des seuls auteurs de guides pratiques. Elles semblent partagées par l'ensemble de la communauté télématique.

41 On peut alors - air connu - déplorer la faible sensibilité aux sciences sociales des télématiciens supposés être recrutés parmi des informaticiens polarisés sur le niveau technologique de fonctionnement de leurs machines. En ce qui me concerne je préférerais inverser la question et me demander plutôt si cette lacune n'est pas la trace d'une autre lacune localisée dans nos propres recherches en Infocom. N'y a-t-il pas dans le mode de fonctionnement des minitels un type de relation à l'écrit et plus généralement de relation à la production de sens qui entrave la conceptualisation des appropriations. Auquel cas au lieu d'appliquer aux minitels des modes de pensée empruntés à l'appropriation de l'écrit, il faudrait isoler cette singularité des minitels de façon à renouveler le questionnement sur les techniques d'inscription.

\section{Télématique et autorat éditorial.}

L'articulation essentielle de cet impensé théorique dont la télématique grand public permet de prendre conscience me paraît tenir à la question de l'autorat, son renouvellement moderne et les problèmes déontologiques issus de ce renouvellement.

En effet la première conséquence d'une problématique d'appropriation est que, se mettant du côté du lecteur ou de l'usager, elle globalise, dans un premier temps, l'offre qui se propose à elle. Caractères alphabétiques, organisation spatiale, ergonomie... tout fait sens. Tout est à déchiffrer et donc à classer. Et dans un deuxième temps l'appropriateur désenmêle les énoncés. Il cherche l'énoncé principal, il essaye de séparer le texte du contexte. Or dans les techniques d'inscription modernes, et pas seulement la télématique, une partie de la production de sens se déplace du texte vers le contexte.

J'ai montré à propos du Hitchcock/Truffaut, ce livre en forme de film que François Truffaut (1983) a consacré à son ami Hitchcock, qu'un auteur, s'inspirant des acquis de la mise en page du journalisme moderne, pouvait déplacer sa production de sens du plan de la langue vers l'organisation iconique et graphique de l'espace imprimé. De ce fait, il y a 
une sorte de diversification de l'autorat. A celui, traditionnel, des énonciers langagiers s'ajoute celui de la mise en page qui est une mise en scène (Poulle 1991).

Et encore dans cet exemple, la question est simplifiée du fait que la même personne physique (François Truffaut) réunit les deux casquettes. Mais dans le cas de la télématique cette diversification de l'autorat est un véritable éclatement. L'usager qui consulte un service est certes confronté à un auteur identifié et doté d'une responsabilité éditoriale, un service qui a un nom dans l'annuaire et un code d'accès. Mais la production de sens de ce service est entièrement captive de très nombreux facteurs techniques qui ne sont ni identifiés, ni identifiables. C'est Transpac, c'est la logique informatique du serveur qui loge le service, c'est le logiciel avec lequel le service a été conçu. En d'autres termes la télématique, bien qu'elle use de caractères alphabétiques, opère en rupture avec les techniques d'inscription traditionnelles (écriture, imprimerie). Chez celles-ci aussi la dimension technique est productrice de sens mais pas au point d'aspirer à l'autorat. Et télématique, et dans son état actuel, l'autorat en amont du texte semble l'emporter sur l'autorat traditionnel, l'autorat langagier.

Le phénomène est d'autant plus troublant que, comme on le disait plus haut, le mode de communication instaurée par le minitel s'apparente à celui de la conversation. Il y a simulation d'une conversation. Et cette simulation a des conséquences déontologiques importantes.

47 Autorat et déontologie ont en effet partie liée dans la mesure où pour lutter contre les manipulations des énoncés qu'autorise le système des langues et qu'a dénoncé, en son temps, Ésope, nous avons pris l'habitude de fournir à nos locuteurs des gages de la véracité de nos énoncés. Goody (1986) a fait remarquer que l'une des conséquences de l'apparition de l'écriture est « la perte de la présence physique du locuteur ». Et donc du gage de vérité qu'apporte cette présence. Cette perte de la présence physique est compensée par les différentes techniques d'inscription grâce à un recours à la rhétorique qu'on ne détaillera pas ici. L'essentiel est que cette nécessité d'une garantie fasse l'objet d'un déplacement sur plusieurs plans simultanément : renforcement de la rhétorique, apparition d'une magistrature éditoriale, codification cognitive des matériaux sur lesquels s'inscrivent les inscriptions.

48 Dans le cas de la télématique le "retour de la personne " s'effectue dans le même temps qu'il y a absence de la personne sur le plan de la responsabilité des énoncés. L'autorat n'est pas seulement éclaté entre plusieurs énonciateurs. Il est réparti entre des énonciateurs se présentant comme tels et d'autres que nos habitudes mentales ne sont pas accoutumées à voir comme porteurs d'énonciations.

Davantage encore que les autres techniques d'inscription, le minitel assigne à l'activité éditoriale un statut d'autorat. Et surtout aux dimensions techniques ou technologiques de cette activité. Mais l'institution télématique ne pense pas pour autant cet autorat. Elle envisage certaines conséquences juridiques (responsabilités des gérants de service) mais ne va pas jusqu'à se penser comme mise en scène.

50 Cette ambiguïté de l'autorat explique sans doute en partie la pusillanimité des rédacteurs de guides pratiques. Face à une machine qui produit du sens mais selon des lois non connues, ils ont préféré se retirer sur ce qu'ils connaissaient : le fonctionnement technique. S'avancer dans l'évaluation des appropriations lectorielles c'est être capable de faire la part entre les dysfonctionnements accidentels, imputables à la machine (comme l'est la « coquille » en matière d'imprimerie) et les dysfonctionnements signés, 
ceux dont quelqu'un peut rendre compte. Or non seulement les moyens de cette élucidation manquent mais, du fait du système d'acte d'achat adopté, le flou arrange tout le monde : gestionnaires de réseaux, logeurs de services et rédacteurs de pages-écrans. Dans la représentation sociale, la relation est présentée comme acte technique davantage que comme un acte communicationnel.

51 A y réfléchir, la recherche en Inforcom aurait tout intérêt à élucider ce flou. Loin d'être un cas d'espèce, l'imbroglio télématique anticipe sur l'évolution des médias. Ceux-ci (presse, télévision, édition informatique) font de plus en plus des offres dans lesquelles la matérialisation technologique des énoncés intervient comme production de sens. Face à ces offres globales l'usager doit apprendre à naviguer, s'approprier du sens même s'il n'est pas verbalisé ou verbalisable. Et la recherche doit se donner les moyens d'évaluer cette appropriation.

\section{BIBLIOGRAPHIE}

FUTUR SIMPLE, (1987), Les services videotex universitaires, Paris. DBMIST/DGT.

Goody, J., (1986), La raison graphique. La domestication de la pensée sauvage, Éditions de Minuit, Paris.

Guéry, L., (1983), L'écriture et la mise en page télématique, Paris, CFPJ.

PoulIe, F., (1991), L'édition video et le modèle du livre, Grenoble. H.D.T. en cours.

Truffaut, F., (1983), HITCHCOCK/TRUFFAUT Édition définitive, Ramsay, Paris.

\section{NOTES}

1. Et comme chacun sait c'est là le moindre de ses défauts.

2. Notamment parce que ces rédacteurs sont, par ailleurs, des chercheurs professionnels. On y revient plus loin.

\section{RÉSUMÉS}

L'auteur examine et compare six " guides pratiques pour minitel » vendus en librairies. Ces objets ont un statut ambigu. Ils postulent un usager soit débile (comment brancher un minitel) soit en quête d'informations très pointues et pas très utiles pour la vie courante (la structure de Transpac). Par contre, ils ne renseignent pas le lecteur sur ce que celui-ci aimerait logiquement connaître (des conseils pour une démarche d'appropriation). C'est d'autant plus singulier que, 
comparé à d'autres médias, le mode de facturation du minitel rend décisive cette question de l'appropriation.

François Poulle formule l'hypothèse d'une publicité clandestine par Guides pratiques interposés. Il l'abandonne faute de preuves. Il examine alors si l'origine de cette ambiguïté des guides pratiques ne doit pas être recherchée dans les lacunes de la recherche théorique en inforcom. La réflexion sur l'autorat et notamment l'autorat éditorial serait en retard par rapport au développement des machines à communiquer.

The writer examines and compares six « Practical Guides for Videotex » sold in bookshops. These guides have an equivocal character. They suppose either a dull-minded reader (how to plug in a videotex) or a reader looking for very specialized information which are not useful in common life (the videotex structure). On the other hand they don't inform the reader on the points they would reasonably want to know such as the way of using it cleverly and fast enough to make it economical. If we compare it with other media it is all the more strange as the expenses incurred make it necessary to have the ability of using it.

F. Poulle makes the assumption that it is perhaps a clandestine publicity through the intermediary of practical guides. He gives it up for want of evidence. Then he studies whether the origin of that ambiguity of practical guides should not be looked for in the gaps of theorical research in Information and Communication sciences. The speculation about the links between authorship, publishing and, especially editorship seems to be late compared to the development of communication machines.

\section{INDEX}

Mots-clés : manuel, usager, étude d'usage, usage des TIC, besoin d'information, Minitel, télématique

Keywords : handbook, user, usage study, information need, videotext, telematics, ICT usage

\section{AUTEUR}

\section{FRANÇOIS POULLE}

François Poulle, Université Paris XII. 